\title{
Screening for Symptoms of Anxiety and Depression in Patients treated with renal replacement therapy Utility of the Edmonton Symptom Assessment System- Revised
}

\section{Evan Tang}

University Health Network

\section{Sumaya Dano}

University Health Network

\section{Nathaniel Edwards}

University Health Network

\section{Sara Macanovic}

University Health Network

Heather Ford

University Health Network

\section{Susan Bartlett}

McGill University

\section{Doris Howell}

Princess Margaret Hospital: Princess Margaret Hospital Cancer Centre

Madeline Li

Princess Margaret Hospital: Princess Margaret Hospital Cancer Centre

\section{Marta Novak}

University Health Network

Istvan Mucsi ( $\square$ istvan.mucsi@utoronto.ca )

University Health Network https://orcid.org/0000-0002-4781-4699

\section{Research Article}

Keywords: Edmonton Symptom Assessment System-revised (ESASr), renal replacement therapy, anxiety, depression, Patient Health Questionnaire-9 (PHQ-9)

Posted Date: June 7th, 2021

DOI: https://doi.org/10.21203/rs.3.rs-190783/v1

License: (c) (i) This work is licensed under a Creative Commons Attribution 4.0 International License. Read Full License

Version of Record: A version of this preprint was published at Quality of Life Research on June 17th, 2021. See the published version at https://doi.org/10.1007/s11136-021-02910-5. 


\section{Abstract}

Purpose: The Edmonton Symptom Assessment System-revised (ESASr) is widely used in clinical oncology to screen for physical and emotional symptoms. The performance of the anxiety and depression items (ESASr-A and ESASr-D, respectively) as screening tools has not been evaluated in patients treated with renal replacement therapy.

Methods: Kidney transplant recipients and patients on dialysis were recruited in Toronto. Patients were classified as having moderate/severe depression and anxiety symptoms using the established cut-off score of $\geq 10$ on the Patient Health Questionnaire-9 (PHQ-9) and the General Anxiety Disorder-7 (GAD-7) questionnaires.

Results: This study included 931 participants; $62 \%$ male, mean age (SD) 55(16), and 52\% White. All participants completed ESASr, however only 748 participants completed PHQ-9 and 769 participants completed GAD-7. Correlation between ESASr item scores and legacy scores were moderately strong (ESASr-D/PHQ-9: 0.61; ESASrA/GAD-7: 0.64). We found good discrimination for moderate/severe depression and anxiety (area under the receiver operating characteristics curve [95\%Cl]: ESASr-D 0.82 [0.78-0.86]; ESASr-A 0.87 [0.82, 0.92]). The cut-off $\geq 2$ for ESASr-D (Sensitivity $=0.76$; Specificity $=0.77$; Likelihood Ratio(LR) $+=3.29 ;$ LR- $=0.31$ ) and $\geq 4$ for ESASr$A$ (Sensitivity $=0.75$; Specificity $=0.87 ; \mathrm{LR}+=5.76 ; \mathrm{LR}-=0.29$ ) had the best combination of measurement characteristics.

Conclusion: The identified ESASr-D and ESASr-A cut-off scores may be used to rule out patients without emotional distress with few false negatives. However, the low sensitivity identified in our analysis suggests that neither ESASrD or ESASr-A are acceptable as standalone screening tools.

\section{Introduction}

Patients with end stage kidney disease (ESKD) who are treated with renal replacement therapy (RRT - dialysis or kidney transplant) experience substantial physical and emotional symptom burden [1-3]. Depression and anxiety are common symptoms that are associated with impaired health-related quality of life (HRQOL), suboptimal treatment adherence, and poor clinical outcomes [1, 4-10]. Furthermore, patients with kidney failure prioritized reducing symptoms, improving psychosocial well-being and HRQL[11]. Despite this, however, depression and anxiety among patients treated with RRT are often unrecognized and undertreated $[12,13]$.

Valid, reliable patient reported outcome measures (PROMs) that have good discrimination may be used to screen for emotional symptoms[14-17]. Recent studies considered the Edmonton Symptom Assessment System (ESAS) to routinely measure symptoms experienced by patients on dialysis $[18,19]$. Routine symptom assessment with ESAS improved awareness of symptom burden among providers and patients, particularly for psychosocial symptoms but it had minimal impact on symptom management. One potential barrier to efficiently use ESAS may be the lack of standardized triggers for when to respond to results[18].

The ESAS has been developed for symptom assessment in patients with cancer and has been translated and validated in several languages [20, 21]. It assesses nine symptoms using a single rating scale item for each symptom. The revised tool (ESASr) specifies timeframe and definitions of symptoms [20, 22]. ESASr poses minimal burden to patients [21] and is used to monitor symptom burden in patients with cancer in Ontario [23-25] and in other jurisdictions [26-28]. The reliability and validity of ESASr have been assessed in patients on RRT [29-31].

In this study, we assessed the discrimination and screening performance of ESASr- depression (ESASr-D) and ESASranxiety (ESASr-A) items in relation to the Patient Health Questionnaire-9 (PHQ-9) (depression) and the Generalized 
Anxiety Disorder-7 (GAD-7) (anxiety) in patients treated with RRT. We also assess the precision of potential cut off scores to identify patients with moderate/severe symptoms of depression and anxiety who may benefit from further multidimensional assessment.

\section{Methods}

\section{Study Design}

This is a secondary analysis of data collected in a multicenter, cross-sectional study drawn from the COmprehensive Psychosocial REsearch Data System (Co-PreDS-CKD), a research database that we established (REB\#17-5916). We used data from patients on maintenance dialysis and KTRs in Toronto, Ontario who completed all ESASr items, PHQ9 and GAD-7 questionnaires at the same time between November 2015 and Dec 2019. All patients included in the studies provided written informed consent. Patients included in the study initiated dialysis treatment at least 90 days or had their kidney transplant a minimum of 30 days prior to enrollment. Exclusion criteria were: insufficient English proficiency, diagnosis of dementia and/or severe acute medical conditions.

Patients were approached during regular clinic visits or dialysis treatment. Participants completed questionnaires on iPads using an electronic data capture system (DAta Driven Outcomes System - DADOS, Techna Institute, University Health Network, Toronto). The study questionnaires also included questions about sociodemographic characteristics including age, sex, marital status, education level, income and ethnicity. Clinical data was abstracted from medical records and entered into the database after quality checks and audits. Clinical variables included time since dialysis start or receipt of transplant, blood hemoglobin, serum albumin, and estimated glomerular filtration rate [eGFR]. Glomerular filtration rate (eGFR, in $\mathrm{mL} / \mathrm{min} / 1.73 \mathrm{~m}^{2}$ ) was estimated using the Chronic Kidney Disease Epidemiology Collaboration equation[32]. Comorbid conditions were obtained from medical records to calculate the Charlson Comorbidity Index (CCI)[33].

\section{Questionnaires}

The ESASr assesses nine symptoms (Pain, Tiredness, Drowsiness, Nausea, Lack of Appetite, Shortness of Breath, Depression, Anxiety, and Wellbeing) using a 10-point Likert scale ranging from 0 (no symptom) to 10 (worst possible symptom) [21].

The GAD-7 and PHQ-9 are publicly available screening tools which ask about the extent to which respondents are bothered by symptoms of anxiety and depression, respectively. These questionnaires contain 7 items and 9 items, respectively, with responses rated on a scale of 0-3 (0 "not at all bothered" and 3 "bothered nearly every day"). The total score is a summation of the item scores and ranges from 0-21 (GAD-7) and 0-27 (PHQ-9) [34, 35]. The British Columbia Renal Agency has recommended the use of GAD-7 and PHQ-9 as screening tools in patients with chronic kidney disease [36]. Patients were classified as "anxious" and "depressed" based on a cut-off score of $\geq 10$ on the GAD-7 and PHQ-9, respectively. These cut off scores identify patients with moderate/severe anxiety or depression $[34,35]$.

\section{Statistical Analysis}

Baseline characteristics are presented as frequency, mean, standard deviation, median and interquartile range. Floor and ceiling effects were reported as the proportion of patients with minimum and maximum possible scores, respectively. Criterion validity is assessed though correlations between ESASr-D or ESASr-A and the corresponding 
legacy questionnaire (PHQ-9 or GAD-7) using Spearman's rank sum correlation. Score differences between groups were assessed using Mann-Whitney U test.

Receiver Operating Characteristics (ROC) analyses were conducted to assess the discrimination of ESASr items against moderate/severe depression and anxiety defined by the PHQ-9 or GAD-7 cut-off score $\geq 10$. Test discrimination is characterized by the area under the receiver operating characteristic curve (AUROC), with an AUROC of 0.75 or greater representing good discrimination [37].

We identified cut-off scores to identify "cases" with moderate/severe symptoms for further assessment using Youden's J [38]. The performance of ESASr-D and ESASr-A items was further assessed by calculating sensitivity, specificity, positive and negative predictive value (PPV/NPV), positive and negative likelihood ratio (LR+/LR-). The 95\% confidence interval for optimal cut-off scores identified by the Youden index were reported using bootstrapping techniques with the "cutpt" program in STATA[39].

To further assess pre-test probability and likelihood ratios of the suggested cut-off scores were integrated using Bayesian principles to create a conditional probability plot [40].

All statistical analyses were conducted using STATA 14.0 (StataCorp, College Station, TX).

\section{Results}

\section{Sample Characteristics}

Data from 456 (49\%) KTRs and 475 (51\%) patients on maintenance dialysis were used in this analysis (Figure S1). The mean (SD) age of the entire cohort was 56 (16) years and $60 \%$ of participants were male. The baseline characteristics of the participants are shown in Table 1.

\section{Depression (ESASr-D).}

A total of 748 patients completed both ESAS-r and PHQ-9 ( $n=422$ patients on dialysis, $n=326$ KTRs). Relative to KTRs, patients on dialysis had significantly higher scores on both PHQ-9 (median (IQR) 3 (0-6) vs 5 (2-10), p<0.001) and ESASr-D (Table 2; median (IQR) 0 (0-2) vs 0 (0-3), p=0.001). Based on the PHQ-9 cut-off score of $\geq 10,21 \%$ of the entire cohort had moderate/severe depression, with significantly more patients on dialysis reporting moderate/severe depression compared to KTRs ( $26 \%$ vs $14 \%, p<0.001)$. ESASr-D was moderately correlated with PHQ-9 scores in the entire cohort with Spearman's rho $=0.61, \mathrm{p}<0.001$.

\section{Discrimination and Potential Cut-off score}

In the ROC analysis for the entire cohort of patients on dialysis and KTRs who completed PHQ-9 ( $\mathrm{n}=748)$ good discrimination was noted for ESASr-D regarding moderate/severe depression (Area under ROC=0.82, 95\% Cl: [0.780.86]; Figure 1). Similarly, good discrimination was seen for ESASr-D when the transplant and dialysis cohorts were analyzed separately (Figure S2A and S2B). Based on the Youden's J, the best cut-off score for ESASr-D in the entire cohort was $\geq 2$ (95\%Cl: 0.35-2.65; Sensitivity = 0.76; Specificity = 0.77; LR+ = 3.29; LR- = 0.31; Table 3). The same cut-off score for ESASr-D was identified in both sub-cohorts (dialysis and KTR), with similar NPV and PPV.

Results of the threshold analysis for the entire cohort and the sub-cohorts are shown in Tables 3., S1 and S2, respectively. For the suggested cut-off of ESASr-D $\geq 2$, the NPV (0.92) was high, however, the PPV (0.47) was moderate to low. 
The prevalence of moderate/severe depression is reported to range from 20-40\% in patients with ESKD [41, 42]. Considering a theoretical cohort of 100 patients with an underlying prevalence of moderate/severe depression of $30 \%$, compared to a cut-off score of $\geq 5$, a cut-off of $\geq 2$ identifies less patients correctly, but misses twice as many patients with moderate/severe depression (Figure S3A and S3B).

\section{Post-Test Probability}

Positive and negative post-test probability plots for depression using ESASr-D are shown in Figure S4. At a prevalence of $30 \%$, a positive result on ESASr-D with a cut-off score of $\geq 2$ would result in a post-test probability of depression of $59 \%$. A negative test with a cut-off score of $\geq 2$ would result in a post-test probability of $12 \%$. The results are overall similar in the prevalence range of $20-40 \%$.

\section{Anxiety_(ESASr-A).}

A total of 769 patients completed the ESAS-r and GAD-7 ( $n=440$ patients on dialysis, $n=329$ KTRs). GAD-7 scores were significantly higher for patients on maintenance dialysis vs KTRs (Table 2; median (IQR) 3 (0-6) vs 1 (0-5) for dialysis vs KTRs, respectively; $p=0.002$ ). The median ESASr-A item score, however, was similar for patients on dialysis vs KTR. Based on the GAD-7 cut-off, $13 \%$ of the entire cohort had moderate/severe anxiety which was more frequent in patients on dialysis compared to KTRs (15\% vs $9 \%, p=0.01)$. ESASr-A was moderately correlated with GAD-7 in the entire cohort with Spearman's rho $=0.62, p<0.001$.

\section{Discrimination and Potential Cut-off score}

ESASr-A exhibited good discrimination for moderate/severe anxiety for the entire cohort of patients on dialysis and KTRs who completed GAD-7 ( $n=769$; AUROC: $0.86,95 \% \mathrm{Cl}[0.82,0.90]$; Figure 2). The optimal cut-off for ESASr-A in the entire cohort based on Youden's J was $\geq 4$ (95\%Cl: 2.27-4.73; Sensitivity = 0.75; Specificity = 0.87; LR+ = 5.76; LR= 0.29; Table 4). This cut-off had a high NPV (0.96), but the PPV (0.46) was moderate to low. Similarly, good discrimination was seen for ESASr-A when assessed in the KTRs and dialysis groups separately (Figure S5A and S5B) with the same cut-off score of $\geq 4$ identified in both sub-cohorts (Tables S3 and S4).

The prevalence of anxiety is reported to range from around $10-50 \%$ in patients with ESKD $[7,10]$. Taking a theoretical cohort of 100 patients with an underlying prevalence of moderate/severe anxiety of $30 \%$, compared to a cut-off score of $\geq 5$, a cut-off score of $\geq 4$ identifies more patients correctly, at the expense of somewhat more false negatives (Figure S6A and S6B).

\section{Post-Test Probability}

Positive and negative post-test probability plots of anxiety using ESASr-A are shown in Figure S7. At a prevalence of $30 \%$, a positive result on ESASr-A with a cut-off score of $\geq 4$ would result in a post-test probability of anxiety of $71 \%$. A negative test would result in a post-test probability of $11 \%$. The results were similar across the $10-50 \%$ range of anxiety prevalence.

\section{Discussion}

In this study we assessed the discrimination of the ESASr emotional symptom items relative to widely used legacy tools (PHQ-9 and GAD-7) in patients treated with RRT. Both ESASr-D and ESASr-A have good discrimination.

Potentially clinically useful cut-off score of $\geq 2$ and $\geq 4$ were identified as potential cut-off scores for ESASr-D and 
ESASr-A, respectively. These were similar for patients on dialysis and KTRs, supporting the use of these cut-off scores for patients with ESKD treated with different renal replacement modalities.

Based on the Youden's J, we identified a potential cut-off score of $\geq 2$ for ESASr-D. Notably, this score was also recommended to identify clinically relevant depression in oncology care and in patients on dialysis respectively [4345]. The sensitivity and specificity of this cut-off was similar to what was previously found for other screening tools $[44,46]$. A higher cut off for ESASr-D would have higher specificity, thus reducing the potential burden on the healthcare system associated with unnecessary further assessment of false positives. However, we would miss many more patients with moderate/severe depressive symptoms due to higher proportion of false negatives. Considering the potential negative implications of depression (e.g. impaired HRQOL, non-adherence) we do not suggest using higher cut-off scores.

For ESASr-A, we identified a potential cut-off of $\geq 4$ with better specificity compared to other ultra-short screening tools [46]. Collister et al. have recommended an ESASr-A cut-off of ${ }^{3} 2$ to identify patients with anxiety[44]. In our analysis the ESASr-A cut-off of $\geq 2$ yielded less false negatives but substantially more false positives compared to $\geq 4$. This may increase screening burden, since many more patients would need additional assessment to "clear" patients erroneously identified as having anxiety.

The results of our analyses were similar for both KTR and patients on dialysis. Moreover, the cut-off scores identified performed similarly across the prevalence range reported for depression and anxiety in all patients with ESKD, supporting their use for both for patients on dialysis and KTRs and this may facilitate continuity of care.

The low post-test probability we observed for a negative ESASr-D and ESASr-A score (below potential cut off) in the reported prevalence range for moderate/severe depressive symptoms suggests that a negative ESASr score may be enough to rule out moderate/severe depressive and anxiety symptoms. However, a positive test on ESASr-D and ESASr-A would not increase the post-test probability sufficiently for confident case finding. Thus, positive screening results will need subsequent confirmatory assessment.

The single item ESASr-D and ESASr-A offer may be considered as ultra-brief screening tools for symptoms of depression and anxiety. However, the relatively low sensitivity and specificity of the potential cut off scores identified in our analyses suggests that neither ESASr-D or ESASr-A is acceptable as a standalone screening tool. Even at the lowest cut-off of 1 , we found low sensitivity, similar to what is reported in patients on dialysis and oncology settings $[45,46]$. The overlap in symptoms of emotional distress with other symptoms experienced in patients on RRT may interfere with accurate screening of symptoms of depression and anxiety. Moreover, given the culturally and ethnically diverse patients in our sample, the wording related to symptoms of depression and anxiety in ESASr-D and ESASr-A may have resulted in under-reporting. As a result of the high number of false negatives, many patients who would benefit from further assessment would miss an opportunity discuss options to management their symptoms. In addition, the lack of specificity at low cut-off scores would result in many false positive patients, thus overwhelming the healthcare system with patient who do not need any further assessment for emotional symptoms.

\section{$\underline{\text { Strengths and Limitations }}$}

The strength of our study includes a relatively large sample size, and inclusion of both patients on dialysis and KTRs, making our results more generalizable. Our sample was also ethnically and culturally diverse. The detailed analysis of ESASr-D and ESASr-A cut-off scores also increases confidence in our findings. Use of probability plots 
allows for the comparison of the identified cut-off scores while varying the prevalence rates of depression and anxiety.

One limitation includes the enrollment of a convenience sample of stable patients with ESKD in a Canadian cohort which may limit the generalizability of the results to less stable patients with ESKD. In addition, a significant proportion of the sample (56\%) scored 0 on the ESASr-D scale. This skewed distribution was also seen in other studies using ESAS and does not necessarily present clinically meaningful impact as all scores at the floor level would indicate the absence of symptoms [45, 47,48]. Further work is needed to assess additional properties of ESASr not addressed in this study, such as test-retest reliability and responsiveness. Moreover, the use of selfreported questionnaires instead of clinical diagnostic interviews as "legacy" comparison is an important limitation of the study. This may have introduced a common-method bias, that may have led to inflated estimates of the diagnostic utility.

\section{Conclusion}

In this study among patients on RRT we found strong evidence of criterion validity and good discrimination for ESASr-D and ESASr-A when compared to legacy PROMs. We propose cut-off scores of $\geq 2$ and $\geq 4$ for ESASr-D and ESASr-A, respectively for pre-screening anxiety or depression symptoms in patients on RRT. These cut-off scores screen out many patients that do not need any further assessment for depression and anxiety. However, due to the low sensitivity, ESASr-D and ESASr-A would not be appropriate as standalone screening tools.

\section{Declarations}

Acknowledgment: The authors thank the research students, the participants of the study and the staff at the participating clinics for their valuable contributions.

Funding: The study was supported in part by a grant from the Kidney Foundation of Canada (KFOC190008) and the Canadian Institutes of Health Research (PJT 165915)

Availability of data and material: Data available upon request

Code availability: Not applicable

Authors' Contribution: Research idea and study design: $E T, S D, I M, M N$; Data acquisition: $S M, H F$; Data analysis: $E T$, $S D, N E$. Revision and interpretation: $E T, S D, I M, M N S B, D H, M L$. ET and SD contributed equally to the manuscript. All authors contributed intellectual content during manuscript drafting or revision and accepts accountability for the overall work.

\section{COMPLIANCE WITH ETHICAL STANDARDS}

Conflict of Interest: The authors declare that there are no known conflicts of interest to disclose.

Ethic approval: Approval was obtained from the University Health Network Research Ethics Board (REB\#17-5916). The procedures used in this study adhere to the tenets of the Declaration of Helsinki.

Informed consent: Informed consent was obtained for all individuals in the study. 
1 Shirazian S, Grant CD, Aina O, Mattana J, Khorassani F, Ricardo AC. Depression in Chronic Kidney Disease and End-Stage Renal Disease: Similarities and Differences in Diagnosis, Epidemiology, and Management. Kidney Int Rep 2017; 2: 94-107.

2 Almutary $\mathrm{H}$, Bonner A, Douglas C. Symptom burden in chronic kidney disease: a review of recent literature. $J$ Ren Care 2013; 39: 140-50.

3 Abdel-Kader K, Unruh ML, Weisbord SD. Symptom burden, depression, and quality of life in chronic and endstage kidney disease. Clin J Am Soc Nephrol 2009; 4: 1057-64.

4 Chilcot J, Davenport A, Wellsted D, Firth J, Farrington K. An association between depressive symptoms and survival in incident dialysis patients. Nephrol Dial Transplant 2011; 26: 1628-34.

$5 \quad$ Novak M, Molnar MZ, Szeifert L, Kovacs AZ, Vamos EP, Zoller R, et al. Depressive symptoms and mortality in patients after kidney transplantation: a prospective prevalent cohort study. Psychosom Med 2010; 72: 527-34.

6 El-Majzoub S, Mucsi I, Li M, Moussaoui G, Lipman ML, Looper KJ, et al. Psychosocial Distress and Health Service Utilization in Patients Undergoing Hemodialysis: A Prospective Study. Psychosomatics 2019; 60: 385-92.

7 Cukor D, Coplan J, Brown C, Friedman S, Cromwell-Smith A, Peterson RA, et al. Depression and anxiety in urban hemodialysis patients. Clin J Am Soc Nephrol 2007; 2: 484-90.

8 Cukor D, Rosenthal DS, Jindal RM, Brown CD, Kimmel PL. Depression is an important contributor to low medication adherence in hemodialyzed patients and transplant recipients. Kidney Int 2009; 75: 1223-9.

9 Palmer SC, Vecchio M, Craig JC, Tonelli M, Johnson DW, Nicolucci A, et al. Association between depression and death in people with CKD: a meta-analysis of cohort studies. Am J Kidney Dis 2013; 62: 493-505.

10 Murtagh FE, Addington-Hall J, Higginson IJ. The prevalence of symptoms in end-stage renal disease: a systematic review. Advances in chronic kidney disease 2007; 14: 82-99.

11 Manns B, Hemmelgarn B, Lillie E, Dip SCPG, Cyr A, Gladish M, et al. Setting research priorities for patients on or nearing dialysis. Clin J Am Soc Nephro 2014; 9: 1813 - 21.

12 Weisbord SD, Fried LF, Mor MK, Resnick AL, Unruh ML, Palevsky PM, et al. Renal provider recognition of symptoms in patients on maintenance hemodialysis. Clin J Am Soc Nephrol 2007; 2: 960-7.

13 Jenkinson HC, Hawkins MM, Stiller CA, Winter DL, Marsden HB, Stevens MC. Long-term population-based risks of second malignant neoplasms after childhood cancer in Britain. Br J Cancer 2004; 91: 1905-10.

14 Kroenke K. Enhancing the clinical utility of depression screening. CMAJ 2012; 184: 281-2.

15 Aiyegbusi OL, Kyte D, Cockwell P, Marshall T, Gheorghe A, Keeley T, et al. Measurement properties of patientreported outcome measures (PROMs) used in adult patients with chronic kidney disease: A systematic review. PLoS One 2017; 12: e0179733.

16 Flythe JE, Hilliard T, Castillo G, Ikeler K, Orazi J, Abdel-Rahman E, et al. Symptom Prioritization among Adults Receiving In-Center Hemodialysis: A Mixed Methods Study. Clin J Am Soc Nephro 2018; 13: 735-45. 
17 Flythe JE, Hilliard T, Lumby E, Castillo G, Orazi J, Abdel-Rahman EM, et al. Fostering Innovation in Symptom Management among Hemodialysis Patients: Paths Forward for Insomnia, Muscle Cramps, and Fatigue. Clin J Am Soc Nephro 2019; 14: 150-60.

18 Evans JM, Glazer A, Lum R, Heale E, MacKinnon M, Blake PG, et al. Implementing a Patient-Reported Outcome Measure for Hemodialysis Patients in Routine Clinical Care: Perspectives of Patients and Providers on ESAS-r:Renal. Clin J Am Soc Nephrol Cjasn 2020.

19 Johnson JA, Sayah FA, Buzinski R, Corradetti B, Davison SN, Elliott MJ, et al. A cluster randomized controlled trial for the Evaluation of routinely Measured PATient reported outcomes in HemodialYsis care (EMPATHY): a study protocol. Bmc Health Serv Res 2020; 20: 731.

20 Hui D, Bruera E. The Edmonton Symptom Assessment System 25 Years Later: Past, Present, and Future Developments. J Pain Symptom Manage 2017; 53: 630-43.

21 Bruera E, Kuehn N, Miller MJ, Selmser P, Macmillan K. The Edmonton Symptom Assessment System (ESAS): a simple method for the assessment of palliative care patients. Journal of palliative care 1991; 7: 6-9.

22 Watanabe SM, Nekolaichuk CL, Beaumont C. The Edmonton Symptom Assessment System, a proposed tool for distress screening in cancer patients: development and refinement. Psycho-Oncology 2012; 21: 977-85.

23 Nipp RD, El-Jawahri A, Ruddy M, Fuh C, Temel B, D'Arpino SM, et al. Pilot randomized trial of an electronic symptom monitoring intervention for hospitalized patients with cancer. Ann Oncol 2019; 30: 274-80.

24 Cancer Care Ontario. Interactive Symptom Assessment and Collection (ISAAC) Tool. 2017, Nov, 7.

25 Han B, Wu X, Guo Y. Improvement of fatigue after vitamin D supplementation in kidney transplant recipients. Medicine 2017; 96: e6918.

26 Nieder C, Kampe TA. Frequency and Prognostic Impact of Consistently Low Edmonton Symptom Assessment System Score in the Patients Treated with Palliative Radiotherapy. Cureus 2018; 10: e2032.

27 Hui D, Titus A, Curtis T, Ho-Nguyen VT, Frederickson D, Wray C, et al. Implementation of the Edmonton Symptom Assessment System for Symptom Distress Screening at a Community Cancer Center: A Pilot Program. Oncologist 2017; 22: 995-1001.

28 Savard J, Ivers H. Screening for clinical insomnia in cancer patients with the Edmonton Symptom Assessment System-Revised: a specific sleep item is needed. Support Care Cancer 2019; 27: 3777-83.

29 Dano S, Pokarowski M, Liao B, Tang E, Ekundayo O, Li V, et al. Evaluating symptom burden in kidney transplant recipients: validation of the revised Edmonton Symptom Assessment System for kidney transplant recipients - a single-center, cross-sectional study. Transp/ Int 2020.

30 Davison SN, Jhangri GS, Johnson JA. Longitudinal validation of a modified Edmonton symptom assessment system (ESAS) in haemodialysis patients. Nephrol Dial Transplant 2006; 21: 3189-95.

31 Davison SN, Jhangri GS, Johnson JA. Cross-sectional validity of a modified Edmonton symptom assessment system in dialysis patients: a simple assessment of symptom burden. Kidney Int 2006; 69: 1621-5. 
32 Levey AS, Stevens LA, Schmid CH, Zhang YL, Castro AF, 3rd, Feldman HI, et al. A new equation to estimate glomerular filtration rate. Ann Intern Med 2009; 150: 604-12.

33 Hemmelgarn BR, Manns BJ, Quan H, Ghali WA. Adapting the Charlson Comorbidity Index for use in patients with ESRD. Am J Kidney Dis 2003; 42: 125-32.

34 Spitzer RL, Kroenke K, Williams JB, Lowe B. A brief measure for assessing generalized anxiety disorder: the GAD-7. Arch Intern Med 2006; 166: 1092-7.

35 Kroenke K, Spitzer RL, Williams JBW. The PHQ-9: Validity of a Brief Depression Severity Measure. Journal of General Internal Medicine 2001; 16: 606-13.

36 Committee BRAKC. Depression and anxiety: The role of kidney care clinics. Vancouver: BC Renal Agency. 2015.

37 Alba AC, Agoritsas T, Walsh M, Hanna S, lorio A, Devereaux PJ, et al. Discrimination and Calibration of Clinical Prediction Models: Users' Guides to the Medical Literature. JAMA 2017; 318: 1377-84.

38 Hajian-Tilaki K. Receiver Operating Characteristic (ROC) Curve Analysis for Medical Diagnostic Test Evaluation. Caspian J Intern Med 2013; 4: 627-35.

39 Clayton P. CUTPT: Stata module for empirical estimation of cutpoint for a diagnostic test. 2013.

40 Maceneaney PM, Malone DE. The Meaning of Diagnostic Test Results: A Spreadsheet for Swift Data Analysis. Clinical Radiology 2000; 55: 227-35.

41 Palmer S, Vecchio M, Craig JC, Tonelli M, Johnson DW, Nicolucci A, et al. Prevalence of depression in chronic kidney disease: systematic review and meta-analysis of observational studies. Kidney Int 2013; 84: 179-91.

42 Lopes AA, Bragg J, Young E, Goodkin D, Mapes D, Combe C, et al. Depression as a predictor of mortality and hospitalization among hemodialysis patients in the United States and Europe. Kidney Int 2002; 62: $199-207$.

43 Bagha SM, Macedo A, Jacks LM, Lo C, Zimmermann C, Rodin G, et al. The utility of the Edmonton Symptom Assessment System in screening for anxiety and depression. Eur J Cancer Care (Engl) 2013; 22: 60-9.

44 Collister D, Rodrigues JC, Mazzetti A, Salisbury K, Morosin L, Rabbat C, et al. Single Questions for the Screening of Anxiety and Depression in Hemodialysis. Can J Kidney Health Dis 2019; 6: 2054358118825441.

45 Vignaroli E, Pace EA, Willey J, Palmer JL, Zhang T, Bruera E. The Edmonton Symptom Assessment System as a screening tool for depression and anxiety. J Palliat Med 2006; 9: 296-303.

46 Mitchell AJ. Pooled results from 38 analyses of the accuracy of distress thermometer and other ultra-short methods of detecting cancer-related mood disorders. J Clin Oncol 2007; 25: 4670-81.

47 Chang VT, Hwang SS, Feuerman M. Validation of the Edmonton Symptom Assessment Scale. Cancer 2000; 88: $2164-71$.

48 Salminen E, Clemens KE, Syrjanen K, Salmenoja H. Needs of developing the skills of palliative care at the oncology ward: an audit of symptoms among 203 consecutive cancer patients in Finland. Support Care Cancer 


\section{Tables}

Table 1. Baseline characteristics of the sample

\begin{tabular}{|c|c|c|c|c|}
\hline & $\begin{array}{l}\text { Entire } \\
\text { CohorT } \\
(\mathrm{N}=931)\end{array}$ & $\begin{array}{l}\text { Dialysis } \\
(\mathrm{N}=475)\end{array}$ & $\begin{array}{l}\text { Transplant } \\
(\mathrm{N}=456)\end{array}$ & $P$ value \\
\hline ler [n(\%)male] & $562(60)$ & $289(61)$ & $273(60)$ & 0.76 \\
\hline N Age (SD) & $56(16)$ & $60(14)$ & $51(15)$ & $<0.001$ \\
\hline icity [n(\%)] & & & & $<0.001$ \\
\hline ucasian & $470(53)$ & $198(44)$ & $272(63)$ & \\
\hline ick & $182(21)$ & $135(30)$ & $47(11)$ & \\
\hline ian & $196(22)$ & $103(23)$ & $93(22)$ & \\
\hline her/Unknown & $31(4)$ & $14(3)$ & $17(4)$ & \\
\hline zation [n(\%)more than 12 years of education] & $543(61)$ & $251(55)$ & $292(66)$ & 0.002 \\
\hline tal Status [n(\%)] & & & & $<0.001$ \\
\hline igle & $212(23)$ & $95(21)$ & $117(26)$ & \\
\hline Irried or Common-law & $501(55)$ & $223(48)$ & $278(62)$ & \\
\hline vorced, Widowed, or Separated & $197(22)$ & $143(31)$ & $54(12)$ & \\
\hline \multicolumn{5}{|l|}{ UAL Income IN CAD [n(\%)] } \\
\hline $0,000 /$ Year & $217(33)$ & $152(48)$ & $65(19)$ & $<0.001$ \\
\hline ,000-\$70,000/ Year & $203(31)$ & $93(30)$ & $110(32)$ & \\
\hline $0,000 /$ Year & $238(36)$ & $70(22)$ & $168(49)$ & \\
\hline Ison Comorbidity Index $[\mathrm{n}(\%<3)]$ & $363(44)$ & $131(34)$ & $232(52)$ & $<0.001$ \\
\hline etes [n\% No)] & $568(62)$ & $237(51)$ & $331(73)$ & $<0.001$ \\
\hline n serum Albumin g/L (SD) & $40(6)$ & $37(4)$ & $42(6)$ & $<0.001$ \\
\hline n Hemoglobin G/L (SD) & $118(19)$ & $110(15)$ & $126(20)$ & $<0.001$ \\
\hline $\begin{array}{l}\text { EN DIALYSIS OR TIME } \\
\text { E TRANSPLANT [n(\%>3 years })]\end{array}$ & -- & $414(93)$ & $295(70)$ & -- \\
\hline ety (gad-7) [n(\%) with moderate/severe aNXIety] & $99(13)$ & $68(15)$ & $31(9)$ & 0.01 \\
\hline $\begin{array}{l}\text { ession (phq-9) [n(\%) with moderate/severe } \\
\text { ession] }\end{array}$ & $157(21)$ & $110(26)$ & $47(14)$ & $<0.001$ \\
\hline
\end{tabular}

Abbreviations: SD, Standard Deviation; CAD, Canadian Dollars; PHQ-9, Patient Health Questionnaire-9; GAD-7, Generalized Anxiety Disorder-7

Table 2. Distribution and descriptive characteristic of the depression and anxiety scores

\begin{tabular}{|l|l|l|l|c|c|c}
\hline & $\begin{array}{l}\text { Entire Cohort } \\
\text { Median (IQR) }\end{array}$ & $\begin{array}{l}\text { Dialysis } \\
\text { Median (IQR) }\end{array}$ & $\begin{array}{l}\text { Transplant } \\
\text { Median (IQR) }\end{array}$ & P value & Floor & Ceiling \\
\hline Depression & & & & & & \\
\hline ESASr-D Score & $0(0-2)$ & $0(0-3)$ & $0(0-2)$ & 0.001 & $56 \%$ & $1 \%$ \\
\hline PHQ-9 Score & $4(1-9)$ & $5(2-10)$ & $3(0-6)$ & $<0.001$ & $20 \%$ & $0 \%$ \\
\hline Anxiety & & & & & & \\
\hline ESASr-A Score & $1(0-3)$ & $0(0-4)$ & $1(0-2)$ & 0.32 & $52 \%$ & $1 \%$ \\
\hline GAD-7 Score & $2(0-6)$ & $3(0-6)$ & $1(0-5)$ & 0.002 & $36 \%$ & $0 \%$ \\
\hline
\end{tabular}

Abbreviations: ESASr-D, Edmonton Symptom Assessment System-revised Item; ESASr-A Edmonton Symptom Assessment System-revised Anxiety Item; PHQ-9, Patient Health Questionnaire-9; GAD-7, Generalized Anxiety Disorder-7; SD, Standard Deviation; IQR, Interquartile Range

Table 3. Threshold analysis for ESASr-D scores against moderate /severe depression (PHQ-9 Score $\geq 10$ ) for the Entire Cohort of patients on dialysis and kidney transplant recipients who completed ESASr-D $(n=748)$ 


\begin{tabular}{|c|c|c|c|c|c|c|c|c|c|c|c|c|}
\hline $\begin{array}{c}\text { Cut } \\
\text { off }\end{array}$ & Sensitivity & Specificity & LR+ & LR- & TP & TN & FP & FN & PPV & NPV & $\begin{array}{c}\text { Distance } \\
\text { to }(0,1)\end{array}$ & $\begin{array}{c}\text { Youden's } \\
\text { J }\end{array}$ \\
\hline$>=0$ & $100 \%$ & $0 \%$ & 1.00 & & 157 & 0 & 591 & 0 & 0.21 & - & 1.00 & 0.00 \\
\hline$>=1$ & $83 \%$ & $66 \%$ & 2.45 & 0.26 & 130 & 391 & 200 & 27 & 0.39 & 0.94 & 0.38 & 0.49 \\
\hline$>=2$ & $76 \%$ & $77 \%$ & 3.29 & 0.31 & 119 & 455 & 136 & 38 & 0.47 & 0.92 & 0.33 & 0.53 \\
\hline$>=3$ & $66 \%$ & $84 \%$ & 4.21 & 0.40 & 104 & 498 & 93 & 53 & 0.53 & 0.90 & 0.37 & 0.51 \\
\hline$>=4$ & $60 \%$ & $89 \%$ & 5.44 & 0.45 & 94 & 526 & 65 & 63 & 0.59 & 0.89 & 0.42 & 0.49 \\
\hline$>=5$ & $54 \%$ & $92 \%$ & 6.81 & 0.50 & 85 & 544 & 47 & 72 & 0.64 & 0.88 & 0.47 & 0.46 \\
\hline$>=6$ & $36 \%$ & $95 \%$ & 6.92 & 0.67 & 57 & 560 & 31 & 100 & 0.65 & 0.85 & 0.64 & 0.31 \\
\hline$>=7$ & $29 \%$ & $98 \%$ & 13.32 & 0.72 & 46 & 578 & 13 & 111 & 0.78 & 0.84 & 0.71 & 0.27 \\
\hline$>=8$ & $17 \%$ & $99 \%$ & 20.33 & 0.84 & 27 & 586 & 5 & 130 & 0.84 & 0.82 & 0.83 & 0.16 \\
\hline$>=9$ & $8 \%$ & $100 \%$ & 45.17 & 0.93 & 12 & 590 & 1 & 145 & 0.92 & 0.80 & 0.92 & 0.07 \\
\hline$>=10$ & $4 \%$ & $100 \%$ & 26.35 & 0.96 & 7 & 590 & 1 & 150 & 0.88 & 0.80 & 0.96 & 0.04 \\
\hline
\end{tabular}

Abbreviations: ESASr-D; Edmonton Symptom Assessment System-revised Item; PHQ-9, Patient Health Questionnaire-9; LR, Likelihood ratio; TP, True positive; TN, True negative; FP, False positive; FN, false negative; NPV: Negative Predictive Value; Positive Predictive Value

Table 4. Threshold analysis for ESASr-A scores against moderate / severe anxiety (GAD-7 Score $\geq 10$ ) for the Entire Cohort of patients on dialysis and kidney transplant recipients who completed ESASr-A $(\mathrm{n}=769)$

\begin{tabular}{|c|c|c|c|c|c|c|c|c|c|c|c|c|}
\hline $\begin{array}{c}\text { Cut } \\
\text { off }\end{array}$ & Sensitivity & Specificity & LR+ & LR- & TP & TN & FP & FN & PPV & NPV & $\begin{array}{c}\text { Distance } \\
\text { to }(\mathbf{0 , 1})\end{array}$ & $\begin{array}{c}\text { Youden's } \\
\text { J }\end{array}$ \\
\hline$>=0$ & $100 \%$ & $0 \%$ & 1.00 & & 99 & 0 & 670 & 0 & 0.13 & - & 1.000 & 0.00 \\
\hline$>=1$ & $89 \%$ & $57 \%$ & 2.08 & 0.19 & 88 & 383 & 287 & 11 & 0.23 & 0.97 & 0.443 & 0.46 \\
\hline$>=2$ & $86 \%$ & $71 \%$ & 2.94 & 0.20 & 85 & 474 & 196 & 14 & 0.30 & 0.97 & 0.325 & 0.57 \\
\hline$>=3$ & $79 \%$ & $81 \%$ & 4.22 & 0.26 & 78 & 545 & 125 & 21 & 0.38 & 0.96 & 0.282 & 0.60 \\
\hline$>=4$ & $75 \%$ & $87 \%$ & 5.76 & 0.29 & 74 & 583 & 87 & 25 & 0.46 & 0.96 & 0.284 & 0.618 \\
\hline$>=5$ & $68 \%$ & $91 \%$ & 7.69 & 0.35 & 67 & 611 & 59 & 32 & 0.53 & 0.95 & 0.335 & 0.589 \\
\hline$>=6$ & $49 \%$ & $96 \%$ & 11.05 & 0.53 & 49 & 640 & 30 & 50 & 0.62 & 0.93 & 0.507 & 0.45 \\
\hline$>=7$ & $37 \%$ & $97 \%$ & 13.91 & 0.64 & 37 & 652 & 18 & 62 & 0.67 & 0.91 & 0.627 & 0.35 \\
\hline$>=8$ & $26 \%$ & $99 \%$ & 17.60 & 0.75 & 26 & 660 & 10 & 73 & 0.72 & 0.90 & 0.738 & 0.25 \\
\hline$>=9$ & $13 \%$ & $100 \%$ & 43.99 & 0.87 & 13 & 668 & 2 & 86 & 0.87 & 0.89 & 0.869 & 0.13 \\
\hline$>=10$ & $7 \%$ & $100 \%$ & 23.69 & 0.93 & 7 & 668 & 2 & 92 & 0.78 & 0.88 & 0.929 & 0.07 \\
\hline
\end{tabular}

Abbreviations: ESASr-A; Edmonton Symptom Assessment System-revised Anxiety Item; GAD-7, Generalized Anxiety Disorder-7; LR, Likelihood ratio; TP, True positive; TN, True negative; FP, False positive; FN, false negative; NPV: Negative Predictive Value; Positive Predictive Value

\section{Figures}




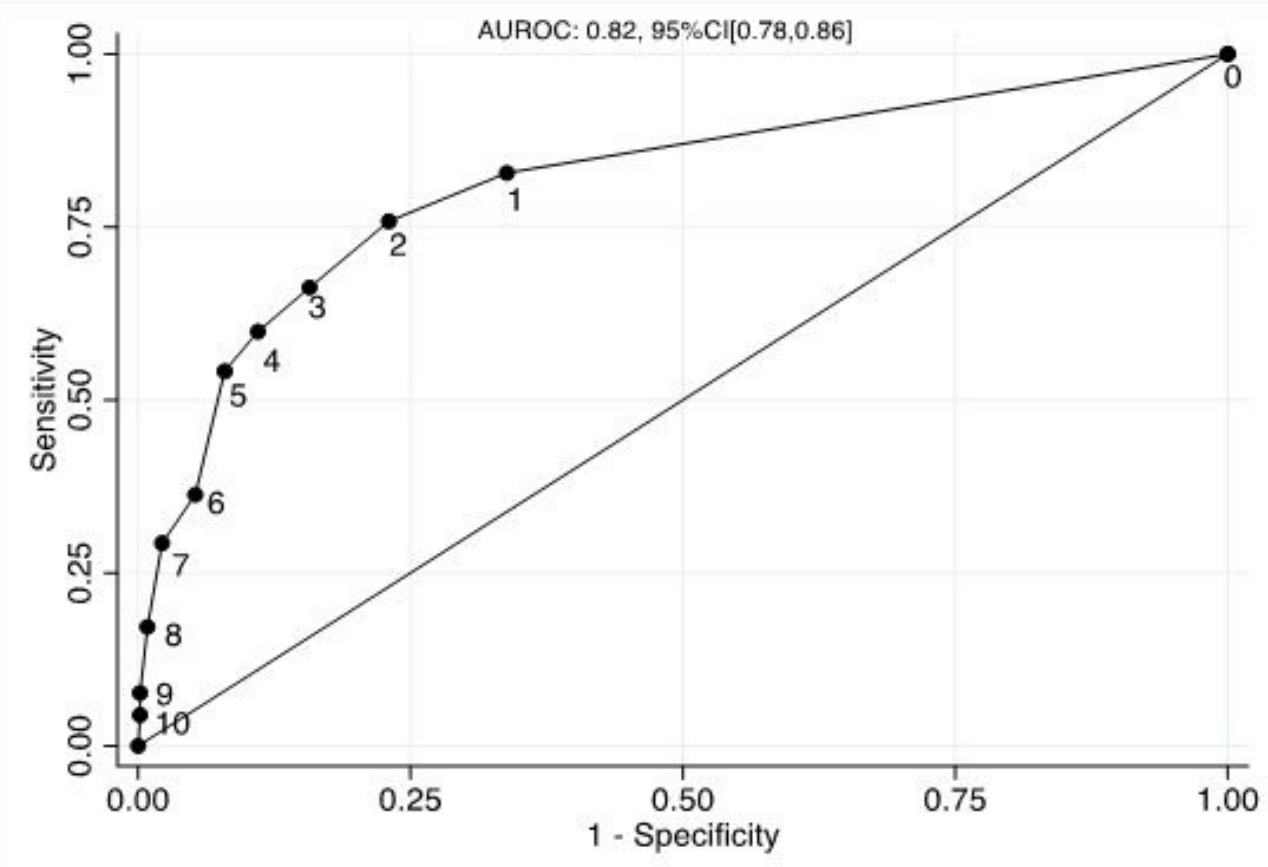

\section{Figure 1}

Receiver operating characteristic curve of Edmonton Symptom Assessment System-revised Depression scores against moderate/severe depression defined by Patient Health Questionnaire- 9 scores $\geq 10$ for the entire cohort of patients on dialysis and kidney transplant recipients who completed the Patient Health Questionnaire-9 $(n=748)$ Abbreviations: AUROC, Area under Receiver operating characteristic curve; Cl, Confidence Interval.

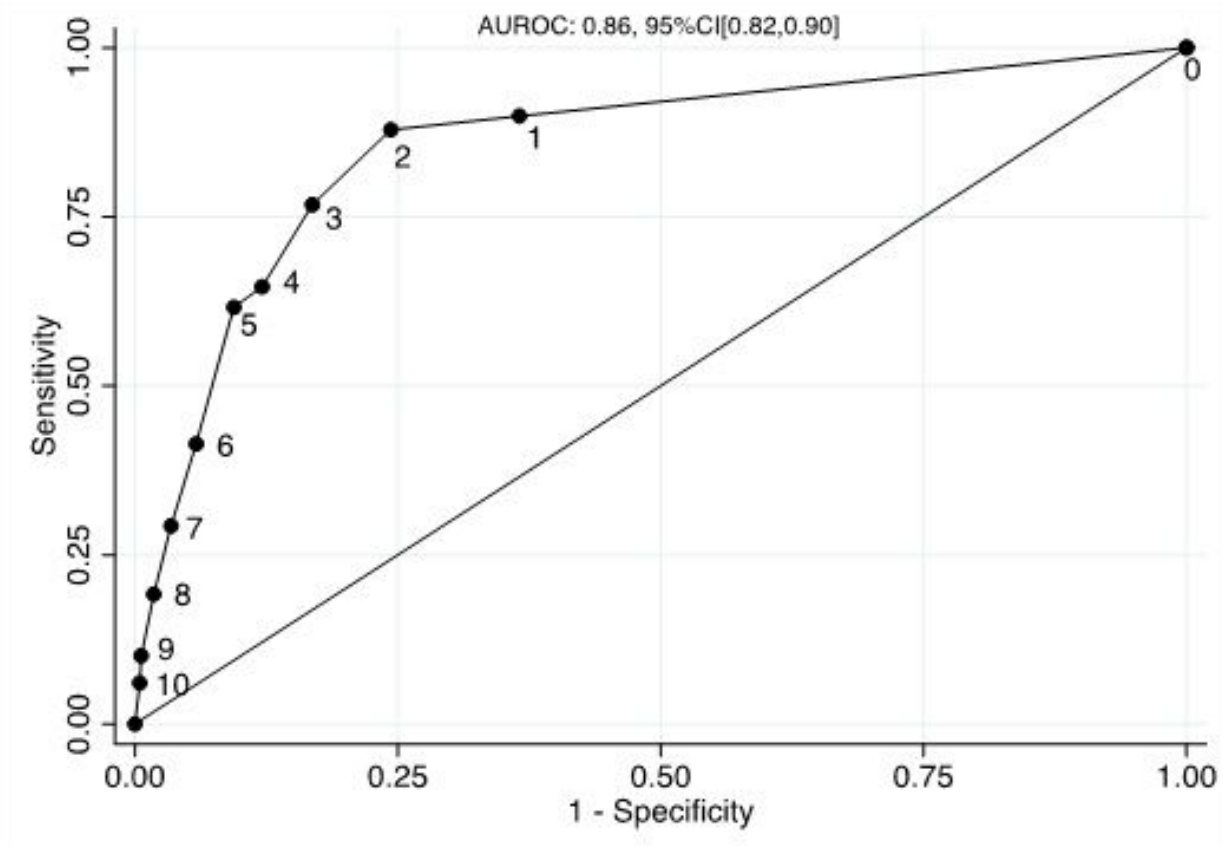

\section{Figure 2}

Receiver operating characteristic curve of Edmonton Symptom Assessment System-revised Anxiety scores against moderate/severe anxiety defined by General Anxiety Disorder -7 scores $\geq 10$ for the entire cohort of patients on 
dialysis and kidney transplant recipients who completed the General Anxiety Disorder -7 ( $\mathrm{n}=769)$ Abbreviations: AUROC, Area under Receiver operating characteristic curve; $\mathrm{Cl}$, Confidence Interval.

\section{Supplementary Files}

This is a list of supplementary files associated with this preprint. Click to download.

- SupplementaryFiguresandTablesQOLClean.docx 